CERN-TH.7418/94

\title{
BEYOND THE INSTANTANEOUS-EXCHANGE APPROXIMATION FOR THREE-DIMENSIONAL QED AT FINITE TEMPERATURE
}

\author{
I.J.R. Aitchison*) \\ Theoretical Physics Division, CERN \\ CH - 1211 Geneva 23
}

\begin{abstract}
Dynamical fermion mass generation is studied in $N$-flavour QED in three dimensions at finite temperature, using the Schwinger-Dyson (S-D) equation. Previous work (in the imaginary-time formalism) approximated the photon propagator $\Delta_{\mu \nu}$ by retaining only the $\mu=\nu=0$ part, at zero frequency ("instantaneous exchange"). Here we calculate the photon propagator to leading order in $1 / N$, in the real-time formalism. Simple approximate forms for the longitudinal $\left(\Pi_{L}\right)$ and transverse $\left(\Pi_{T}\right)$ photon self-energies are proposed. The $\mathrm{S}$-D equation is solved in both the instantaneous and retarded cases. Numerical results for the temperature dependence of the dynamically generated mass $(\Sigma(T))$ are presented, for a range of $N$ values and for different approximate self-energies. The ratio $r=2 \Sigma(T=0) / k_{B} T_{c}$ is generally found to be somewhat reduced when retardation is included, and to depend quite sensitively on the low-momentum behaviour of $\Pi_{L}$ and $\Pi_{T}$. It is concluded that a value $r=6$ is a reasonable estimate in this model.
\end{abstract}

*) Permanent address: University of Oxford, Department of Physics, Theoretical Physics, 1 Keble Road, Oxford OX1 3NP, U.K.

CERN-TH.7418/94

August 1994 


\section{Introduction}

A number of studies have been made of dynamical chiral symmetry breaking (fermion mass generation) in three-dimensional QED [1]-[4] and in four-dimensional QCD [5]-[8] at finite temperature, using the Schwinger-Dyson (gap) equation. This equation is sufficiently complicated that, even though approximations have to be made in deriving it, further approximation are always made in solving it. Given the equation as a plausible starting point, however, it is important to understand to what extent the results obtained are independent of the approximation made in its solution. One can gain insight into this question by trying to isolate, and improve upon, the approximations that have been made.In the case of three-dimensional QED $\left(\mathrm{QED}_{3}\right)$, this approach has been pursued in the series [2]-[4], of which the present paper is a continuation. In Ref. [2], the principal approximations made were as follows: (i) the photon propagator $\Delta_{\mu \nu}$ was calculated to leading order in $1 / N$ in Landau gauge ( $N$ is the number of fermion flavours); (ii) only the $\mu=\nu=0$ part of $\Delta_{\mu \nu}$ was retained; (iii) only the zero-frequency $k_{0}=0$ ("instantaneous exchange") part of $\Delta_{00}$ was retained, retardation effects being neglected; (iv) the fermion self-mass $\Sigma$ was taken to depend on temperature $T$ only, not on momentum $p ;(\mathrm{v})$ fermion wave function renormalization was neglected. The main results were that the dynamically generated fermion mass vanished above a certain critical temperature $T_{c}$, and that the ratio $r=2 \Sigma(T=0) / k_{B} T_{c}$ was of order 10 , in contrast to the value $r=3.54$ characteristic of BCS superconductors. In Ref. [3], approximations (i), (ii), (iii) and (v) were kept, but $\Sigma$ was allowed to depend on $p$ as well as on $T$. In this case, as at zero temperature [9], it was found that $\Sigma(p=0)$ vanished for $N$ greater than a critical $N_{c}$; here, however $N_{c}$ was dependent upon $T$, and a phase diagram of $\mathrm{QED}_{3}$ in the $N-T$ plane was presented. The ratio $r=2 \Sigma(p=0, T=0) / k_{B} T_{c}$ remained of order 10 , approximately independent of $N$. In Ref. [4], an attempt was made to drop approximation (v), as well as (iv), and include the effect of wave function renormalization as well as that of momentum-dependence in $\Sigma$, in view of the relevance of approximation (v) to the existence of a critial $N_{c}$ at $T=0$ [10]. Approximations (ii) and (iii) were again retained, and indeed somewhat extended in the sense that the frequency dependence of the kinematical factor in the kernel of the equation for the wave function renormalization constant $(Z)$ was also ignored. It was found, however, that this led to an unphysical result, namely $Z>1$, even as $T$ approached zero. A modified equation was therefore proposed, in a somewhat ad hoc manner, which gave $Z<1$ and sensible low $T$ limit. A phase diagram was obtained which was very similar to that of [3], and $r$ remained essentially unchanged at about 10 .

It therefore seems that $r \approx 10$ is a rather robust feature of dynamical mass generation in $\mathrm{QED}_{3}$ at $T \neq 0$. The origin of this large value (relative to the 3.54 of BCS theory) was traced in Ref. [2] to the thermal screening associated with the appearance of a (temperature-dependent) photon mass

$$
\Pi_{00}^{1 / 2}\left(p_{0}=0,|p| \rightarrow 0, T\right)=\left[2 \alpha k_{B} T \ln 2 / \pi\right]^{1 / 2},
$$

where $\alpha \equiv N e^{2}$ and $\Pi_{\mu \nu}$ is the vacuum polarization tensor. A similar increase of $r$ from about 3.5 to about 9 was also obtained in Ref. [8] for the case of QCD at $T \neq 0$, when an analogous gluon mass was included. For $\mathrm{QED}_{3}$, a large $r$ has some potential phenomenological significance, since there are indications that the layered $\mathrm{CuO}$ superconductors have values of 
$r$ larger than the BCS value [11],[12], which suggests that the pairing mechanism may arise from a gauge interaction similar to that in $\mathrm{QED}_{3}$, as has been proposed in [13] and elaborated further in [14].

Nevertheless, one must note that Refs. [3] and [4] both retained approximations (ii) and (iii) of [2], as well as the large $N$ approximation (i). (Of the QCD calculations referred to above, Ref. [8] - also in Landau gauge - approximated the gluon self-energies $\Pi_{T}$ and $\Pi_{L}$ by their values at $p^{2}=0$, while Refs. [6] and [7] - in Coulomb gauge - neglected retardation in the transverse gluon potential.) As regards approximation (ii), it was found in [2] that the transverse part of the photon propagator remained massless in the instantaneous limit, which would lead to infrared divergences. Partly for this reason, and partly in order to maintain a close correspondence with static potential theory, only the $\Delta_{00}$ component of $\Delta_{\mu \nu}$ was kept. It is clearly of interest to learn how a more complete propagator might affect the value of $r$.

There are also reasons for querying the reliability of approximation (iii), in this context. The calculations of [2]-[4] were all done using the imaginary time formalism, in which boson (fermion) energies are discrete even (odd) multiples of $i \pi / \beta\left(\beta=1 / k_{B} T\right)$. At sufficiently high temperature it may be a good approximation to retain only the $n=0$ (zero frequency) component of the bosonic function $\Pi_{00}$, since the energy intervals between successive multiples are then large. But chiral symmetry breaking is expected to be a low temperature phenomenon: indeed, the critical $T_{c}$ 's of [2]-[4] are always substantially less than the natural mass scale $\alpha$. Moreover, as $T$ approaches zero, it is clear that all frequency components should be included, so that retaining only the $n=0$ component in $\Pi_{00}$ inevitably means that one cannot recover the well-studied zero-temperature case [9], [10], [15], [16] as $T \rightarrow 0$. Finally, the problem encountered with $Z$ in [4] seemed to be due to the "dimensional reduction" associated with the $n=0$ truncation, which commonly occurs in the high (rather than low) $T$ limit.

The purpose of the present paper is therefore to examine the effect of approximations (ii) and (iii) on dynamical chiral symmetry breaking in $\mathrm{QED}_{3}$ at $T \neq 0$, and specifically their effect on the ratio $r$. While it would clearly be desirable to perform a more or less complete calculation, and relax approximations (ii) and (ii) simultaneously with (iv) and (v) (especially in view of the problem with $Z$ in [4]), we shall not be so ambitious here. Instead, we shall retain approximation (i), (iv) and (v), which means that we shall essentially study the effect of relaxing (ii) and (iii) in simpler models of the type studied in [2].

As mentioned above, Refs. [2]-[4] all used the imaginary time formalism with discrete imaginary energies. To go beyond the instantaneous approximation (iii) in this formulation would require the solution of an increasingly large number (as $T \rightarrow 0$ ) of coupled gap equations for the different energy components [see Eq. (2.51) of [14]]. While this may be a possible approach, it seems more natural to work with continuous energies. These may of course be reached by analytic continuation from the discrete case, but there are subtleties in this procedure which make it simpler to use the real-time formalism from the beginning (this also contains the $T=0$ limit very naturally). This was also the approach followed in the early study [1], but it is not possible to make a direct and systematic comparison between the results of [1] and those of [2], for example, since as we shall see below a number of rather drastic approximations were made 
in [1]. In Section 2, we set up the S-D equation using the real time formalism, and calculate the photon propagator to leading order in $1 / N$, in Landau gauge. We consider some simple approximate expressions for the self-energies $\Pi_{L}$ and $\Pi_{T}$. In Section 3, we compare solutions of the S-D equation in which various approximate forms for $\Pi_{L}$ and $\Pi_{T}$ are taken, and in which the instantaneous approximation to $\Pi_{L}$ and $\Pi_{T}$ is or is not made. Our main conclusion is that $r$ is quite sensitive to approximations (ii) and (iii), and that a value in the region of 6 is a more reasonable estimate.

\section{S-D equation and photon propagator at $T>0$}

To leading order in $1 / N$, all radiative corrections are determined by one-loop graphs, as at $T=0$ [15], so that as observed in [8] the field-doubling of the real-time formalism [17] plays no direct role and we obtain the corect answer [18] using the "naive" old real-time approach [19]. Making approximation ( $\mathrm{v}$ ) of the previous section, and working in the rest frame of the heat bath, the $\mathrm{S}-\mathrm{D}$ equation for the fermion self-mass $\Sigma(p, \beta)$ is then

$$
\Sigma(p, \beta)=\frac{i e^{2}}{4} \operatorname{Tr} \gamma^{\mu} \gamma^{\nu} \int \frac{d^{3} k}{(2 \pi)^{3}} \Delta_{\mu \nu}(q, \beta) S_{F}(k, \beta),
$$

where $q=p-k$. In (2), the fermion propagator is

$$
S_{F}(k, \beta)=(\not k+\Sigma(k, \beta)) \Delta_{f}(k, \Sigma(k, \beta), \beta)
$$

and the photon propagator in Landau gauge is

$$
\Delta_{\mu \nu}(q, \beta)=-P_{\mu \nu} \Delta_{b}\left(q, \Pi_{T}^{1 / 2}(q, \beta), \beta\right)-Q_{\mu \nu} \Delta_{b}\left(q, \Pi_{L}^{1 / 2}(q, \beta), \beta\right)
$$

where the explicit forms of the transverse $\left(P_{\mu \nu}\right)$ and longitudinal $\left(Q_{\mu \nu}\right)$ projection operators are given in Ref. [20], and where

$$
\Delta_{b, f}(k, m, \beta)=\frac{1}{k^{2}-m^{2}+i \epsilon}-\frac{2 \pi i \delta\left(k^{2}-m^{2}\right) \eta_{b, f}}{e^{\beta\left|k_{0}\right|}-\eta_{b, f}}
$$

with $\eta_{b}=+1$ and $\eta_{f}=-1$. The $\gamma$-matrices are $4 \times 4$, corresponding to a continuous chiral symmetry in the original (massless) $\mathrm{QED}_{3}$ Lagrangian. Making approximation (iv) in the form $\Sigma(p, \beta) \approx \Sigma(0, \beta)$ we obtain

$$
1=\frac{-i \alpha}{N} \int \frac{d^{3} k}{(2 \pi)^{3}}\left[\Delta_{b}\left(k, \Pi_{T}^{1 / 2}(k, \beta), \beta\right)+\Delta_{b}\left(k, \Pi_{L}^{1 / 2}(k, \beta), \beta\right)\right] \Delta_{f}(k, \Sigma, \beta),
$$

where we now suppress the remaining argument $\beta$ of $\Sigma$, and we have used

$$
P_{\mu}^{\mu}=Q_{\mu}^{\mu}=1
$$

as appropriate to $2+1$ dimensions (in $3+1$, the transverse part in (6) would appear with a factor 2). 
To proceed further with (6), we need expressions for the self-energies $\Pi_{T}$ and $\Pi_{L}$. These are related to the vacuum polarization tensor $\Pi_{\mu \nu}$ by [20]:

$$
\Pi_{\mu \nu}(k, \beta)=\Pi_{T}(k, \beta) P_{\mu \nu}+\Pi_{L}(k, \beta) Q_{\mu \nu},
$$

where

$$
P_{\mu \nu}+Q_{\mu \nu}=g_{\mu \nu}-\frac{k_{\mu} k_{\nu}}{k^{2}} .
$$

Adapting [20] to 2+1-dimensions we find [1]

$$
\Pi_{L}(k, \beta)=\left(1-\frac{k_{0}^{2}}{k^{2}}\right) \Pi_{00}(k, \beta),
$$

and

$$
\Pi_{T}(k, \beta)=\Pi_{\mu}^{\mu}(k, \beta)-\Pi_{L}(k, \beta) .
$$

For reference, note that at $T=0$ we write

$$
\Pi_{\mu \nu}(k, \infty) \equiv \Pi_{\mu \nu}(k)=\Pi\left(k^{2}\right)\left(g_{\mu \nu}-\frac{k_{\mu} k_{\nu}}{k^{2}}\right),
$$

so that

$$
\Pi_{L}(k, \infty)=\Pi\left(k^{2}\right)=\Pi_{T}(k, \infty),
$$

and we recover from (6) the standard zero-temperature equation of this model (see [15], or Eq. (14) of [2], for example). At finite $T$, it is convenient to separate out the zero-temperature parts, and write

$$
\begin{aligned}
\Pi_{T}(k, \beta) & =\Pi\left(k^{2}\right)+\Pi_{T}^{\prime}(k, \beta), \\
\Pi_{L}(k, \beta) & =\Pi\left(k^{2}\right)+\Pi_{L}^{\prime}(k, \beta), \\
\Pi_{\mu \nu}(k, \beta) & =\Pi_{\mu \nu}(k)+\Pi_{\mu \nu}^{\prime}(k, \beta),
\end{aligned}
$$

where $\Pi_{L}^{\prime}$ and $\Pi_{T}^{\prime}$ are given by $(10)$ and $(11)$ with $\Pi_{\mu \nu}$ replaced by $\Pi_{\mu \nu}^{\prime}$. Finally, according to approximation (i), $\Pi_{\mu \nu}$ is given by the one-loop expression

$$
\Pi_{\mu \nu}(k, \beta)=i \alpha \int \frac{d^{3} p}{(2 \pi)^{3}} \operatorname{Tr}\left\{\gamma_{\mu} S_{F}(p+k, \beta) \gamma_{\nu} S_{F}(p, \beta)\right\}
$$

Turning then to the evaluation of (17), we first note that the term corresponding to the product of the $\delta$-functions in the $S_{F}$ 's [cf. (3) and (5)] contributes only to the imaginary part of $\Pi_{\mu \nu}$, which we shall neglect. Next, the product of the $T$-independent parts of the $S_{F}$ 's yields

$$
\Pi\left(k^{2}\right)=\frac{\alpha \Sigma}{2 \pi}-\frac{\alpha}{8 \pi \sqrt{k^{2}}}\left(k^{2}+4 \Sigma^{2}\right) \ln \left(\frac{2 \Sigma+\sqrt{k^{2}}}{2 \Sigma-\sqrt{k^{2}}}\right),
$$

for $4 \Sigma^{2}>k^{2}>0$, and $[14]$

$$
\Pi\left(k^{2}\right)=\frac{\alpha \Sigma}{2 \pi}-\frac{\alpha}{4 \pi \sqrt{-k^{2}}}\left(k^{2}+4 \Sigma^{2}\right) \cdot \sin ^{-1}\left(\frac{\sqrt{-k^{2}}}{\sqrt{4 \Sigma^{2}-k^{2}}}\right)
$$


for $k^{2}<0$. In most calculations of dynamical mass generation at $T=0$, expression (19) for $\Pi$ has been replaced by its limit as $\Sigma \rightarrow 0$ (namely $\alpha \sqrt{-k^{2}} / 8$ ), since the generated mass $\Sigma$ is in fact much less than the mass scale $\alpha$; inclusion of the fermion mass in $\Pi$ has been found to make little qualitative difference to the results [21]. In the same spirit, the finite temperature calculations of [2] and [3] evaluated the vacuum polarization (in the imaginary time formalism) for massless fermions. Since our intention is to stay as close to the model of [2] as possible, relaxing only approximations (ii) and (iii), we shall generally also consider the massless fermion limit of both $\Pi$ and $\Pi_{\mu \nu}^{\prime}$. However, we shall see that this leads to a problem for some terms in (6).

In the massless limit, then, the expression for $\Pi_{\mu \nu}^{\prime}$ is [1]

$$
\begin{aligned}
\Pi_{\mu \nu}^{\prime}(k, \beta)= & -8 \pi \alpha \int \frac{d^{3} p}{(2 \pi)^{3}}\left[(k+p)_{\mu} p_{\nu}+(k+p)_{\nu} p_{\mu}-g_{\mu \nu}(p+k) \cdot p\right] \times \\
& \left\{\frac{1}{p^{2}+i \epsilon} \cdot \frac{\delta\left((p+k)^{2}\right)}{e^{\left|p_{0}+k_{0}\right| \beta}+1}+\frac{1}{(p+k)^{2}+i \epsilon} \cdot \frac{\delta\left(p^{2}\right)}{e^{\left|p_{0}\right| \beta}+1}\right\} .
\end{aligned}
$$

¿From this, we obtain

$$
\begin{aligned}
\Pi_{\mu}^{\mu^{\prime}}(k, \beta)= & \frac{2 \alpha \ln 2}{\pi \beta}+\frac{\alpha}{2 \pi^{2}}\left(k^{2}-k_{0}^{2}\right)\left\{\int \frac{d^{2} p}{|p|} \cdot \frac{1}{e^{|p| \beta}+1} \cdot\right. \\
& {\left[\frac{1}{\left(k_{0}+|p|\right)^{2}-(k+p)^{2}+i \epsilon}+\left(-k_{0} \text { for } k_{0}\right]\right\} }
\end{aligned}
$$

after performing the $p^{0}$-integration by using the $\delta$-functions, and making an appropriate shift in the momentum integration variable (which is only valid for $\epsilon \neq 0$, otherwise we are manipulating divergent quantities). At this point we observe that we shall ultimately evaluate the $k_{0}$-integration in $(6)$ by rotating into Euclidean space via $k_{0} \rightarrow i k_{3}$. Equation (21) then becomes

$$
\Pi_{\mu}^{\mu^{\prime}}\left(|k|, k_{3}, \beta\right)=\frac{2 \alpha \ln 2}{\pi \beta}-\frac{\alpha}{\pi^{2}}\left(k^{2}+k_{3}^{2}\right) \operatorname{Re} \int \frac{d|p| d \theta}{e^{|p| \beta}+1} \frac{1}{k^{2}+k_{3}^{2}+2|k||p| \cos \theta-2 i k_{3}|p|} .
$$

The angular integral in (22) may be performed following the contour integration method used in a recent study [22] of the odd-parity part of the polarization tensor in $\mathrm{QED}_{3}$ (induced by massive two-component fermions). In this way one finds

$$
\Pi_{\mu}^{\mu^{\prime}}\left(|k|, k_{3}, \beta\right)=\frac{2 \alpha \ln 2}{\pi \beta}-\frac{\alpha}{\pi}\left[2\left(k^{2}+k_{3}^{2}\right)\right]^{1 / 2} \int_{0}^{\infty} \frac{d|p|}{e^{|p| \beta}+1} \frac{\left[k^{2}+k_{3}^{2}-4 p^{2}+l\left(|k|, k_{3},|p|\right)\right]^{1 / 2}}{l\left(|k|, k_{3},|p|\right)}
$$

where

$$
l\left(|k|, k_{3},|p|\right)=\left[\left(k^{2}+k_{3}^{2}-4 p^{2}\right)^{2}+16 p^{2} k_{3}^{2}\right]^{1 / 2} .
$$

Similarly, the quantity $\Pi_{00}^{\prime}$ is given by

$$
\Pi_{00}^{\prime}\left(|k|, k_{3}, \beta\right)=\frac{2 \alpha \ln 2}{\pi \beta}-\frac{\alpha}{\pi} \cdot\left[\frac{2}{\left(k^{2}+k_{3}^{2}\right)}\right]^{1 / 2} \cdot \int \frac{d|p|}{e^{|p| \beta}+1}\left[k^{2}+k_{3}^{2}-4 p^{2}+l\left(|k|, k_{3},|p|\right)\right]^{1 / 2} .
$$


In terms of the above quantities, the functions $\Pi_{L}$ and $\Pi_{T}$ are

$$
\Pi_{L}\left(|k|, k_{3}, \beta\right)=\frac{\alpha}{8}\left[k^{2}+k_{3}^{2}\right]^{1 / 2}+\left(\frac{k^{2}+k_{3}^{2}}{k^{2}}\right) \Pi_{00}^{\prime}\left(|k|, k_{3}, \beta\right)
$$

and

$$
\Pi_{T}\left(|k|, k_{3}, \beta\right)=\frac{\alpha}{8}\left[k^{2}+k_{3}^{2}\right]^{1 / 2}+\Pi_{\mu}^{\mu^{\prime}}-\left(\frac{k^{2}+k_{3}^{2}}{k^{2}}\right) \Pi_{00}^{\prime}\left(|k|, k_{3}, \beta\right) .
$$

These expressions for $\Pi_{T}$ and $\Pi_{L}$ are admittedly cumbersome, and unfortunately no further analytical progress seems to be possible, in general. Nevertheless, a good understanding of their properties is essential before proceeding with (6). First we note that, as usual, the existence of a preferred frame (that of the heat bath) implies that $\Pi_{T}$ and $\Pi_{L}$ are functions of $|k|$ and $k_{3}$ separately, rather than of $k^{2}+k_{3}^{2}$. Secondly, as in an analogous self-energy studied in [22], and in similar four-dimensional cases (see for example [20] and [23], and references cited therein), these functions are not analytic at $|k|=k_{3}=0$, so that their limiting values near the origin depend on the ratio $a=k_{3} /|k|$. Thus we find from (26) and (27) the limits [cf.(1)]

$$
\begin{gathered}
\Pi_{L}\left(|k| \rightarrow 0, k_{3}=0, \beta\right) \longrightarrow \frac{2 \alpha \ln 2}{\pi \beta} \equiv 2 \omega_{P}^{2}, \\
\Pi_{L}\left(|k|=0, k_{3} \rightarrow 0, \beta\right) \longrightarrow \frac{\alpha \ln 2}{\pi \beta} \equiv \omega_{P}^{2}, \\
\Pi_{T}\left(|k| \rightarrow 0, k_{3}=0, \beta\right) \longrightarrow 0, \\
\Pi_{T}\left(|k|=0, k_{3} \rightarrow 0, \beta\right) \longrightarrow \frac{\alpha \ln 2}{\pi \beta} \equiv \omega_{P}^{2} .
\end{gathered}
$$

The right-hand side of $(28 \mathrm{~b})$ and $(28 \mathrm{~d})$ is referred to as the plasmon mass (squared) while (28a) and (28c) correspond to the static case, with screening in case (28a). It may be worth commenting that these limits are quite delicate, and it is essential - for instance - to perform the angular integral in (22) before attempting to take any limits.

We can already make some contact with [2]. That model corresponds to setting $k_{3}=0$ and keeping only $\Delta_{00}$ [approximations (ii) and (iii)]. In this case $P_{00}=0$ and $Q_{00}=1$ [20], and $\Pi_{L}=\Pi_{00}$, so that effectively only $\Pi_{L}$ is retained, having the limit $(28 \mathrm{a})$ in agreement with Eq. (9) of [2] (note that our $\Pi_{00}$ is $-k^{2}$ times the $\Pi_{00}$ of [2]). Complete expressions for the self-energy functions in the imaginary time formalism have been given in [14], but these use a Feynman parametrization of the loop integral, which makes the analytic continuation to continuous energies rather involved [22].

For general $|k|, k_{3}$ and $\beta$, and without making approximations (ii) and (iii), we have to evaluate (26) and (27) numerically and then solve (6). But our present aim is less ambitious. We would like to find simple analytical expressions for $\Pi_{L}$ and $\Pi_{T}$, which are reasonably accurate representations of the exact functions, have the correct zero-temperature limit, and include the particular model of [2] in the instantaneous $\left(k_{3}=0\right)$ limit; we can then do a controlled exploration of retardation effects $\left(k_{3} \neq 0\right)$. For this purpose it is useful to have a qualitative 
picture of the behaviour of the exact $\Pi_{L}, \Pi_{T}$ as given numerically by (26) and (27). This is provided in Figure 1, which shows $\Pi_{L}$ and $\Pi_{T}$ (not to scale) as functions of $|k|$ and $k_{3}$, at fixed $\beta$, on the three planes $a=0\left(k_{3}=0\right), a=1 / 2\left(k_{3}=|k| / 2\right)$ and $a=\infty(|k|=0)$. Only $k_{3}>0$ needs to be considered, as $\Pi_{L}$ and $\Pi_{T}$ are even functions of $k_{3}$. The distance OB is equal to $2 \omega_{P}^{2}[\mathrm{cf} .(28 \mathrm{a})]$, and $\mathrm{OA}$ is equal to $\omega_{P}^{2}$ [cf. (28b) and (28d)]. In the case of $\Pi_{L}$, as $a$ increases the intercept on the vertical axis (i.e., the limit as $k_{3}$ and $|k|$ tend to zero with fixed $a$ ) varies smoothly between $2 \omega_{P}^{2}$ and $\omega_{P}^{2}$; for $\Pi_{T}$ it varies between zero and $\omega_{P}^{2}$. The curves of $\Pi_{L}$ and $\Pi_{T}$ for fixed $a$ are approximately parabolic near the origin, as was found in [3] for $\Pi_{00}$ at $k_{3}=0$ [see Eq. (9) of that reference]. As $T \rightarrow 0$ the curves approach the usual zero-temperature limit, which is the cone $\alpha\left[k^{2}+k_{3}^{2}\right]^{1 / 2} / 8$. In short, what was the same single cone for both $\Pi_{L}$ and $\Pi_{T}$ at zero temperature, has been split at finite temperature into infinitely many curves (depending on $a$ ), and in a way that is asymmetric as between $\Pi_{L}$ and $\Pi_{T}$.

It is not difficult to invent simple functions with the behaviour shown in Fig. 1. We have found that the functions

$$
\Pi_{L}^{a p p}\left(|k|, k_{3}, \beta\right)=\left[\alpha^{2} \frac{\left(k^{2}+k_{3}^{2}\right)}{64}+4 \omega_{P}^{4}\left(1-\frac{1}{\pi} \tan ^{-1} a\right)^{2}\right]^{1 / 2},
$$

and

$$
\Pi_{L}^{a p p}\left(|k|, k_{3}, \beta\right)=\left[\alpha^{2} \frac{\left(k^{2}+k_{3}^{2}\right)}{64}+\left(\frac{2 \omega_{P}^{2}}{\pi} \tan ^{-1} a\right)^{2}\right]^{1 / 2},
$$

provide a generally rather accurate (error less than 10\%) representation of the exact $\Pi_{L}$ and $\Pi_{T}$. No doubt something more elaborate, along the lines of the approximate function introduced in Eq. (9) of [3], could be found - but improvements of a few percent seem to be irrelevant for our purposes. Indeed, we are going to use somewhat cruder approximations even than (29) and $(30)$, both in order to simplify the resolution of $(6)$ and in order to make direct contact with the results of [1] and [2].

The problem with (26) and (27) [and with (29) and (30)] is of course that they spoil threedimensional rotational symmetry among $k$ and $k_{3}$. If the $\Delta_{b}$ 's in $(6)$ did only depend on $k^{2}+k_{3}^{2} \equiv r^{2}$, the integral would become one-dimensional, and the computation would be relatively trivial. Here we shall be content to consider rotationally symmetric approximations to $\Pi_{T}$ and $\Pi_{L}$, which we expect to be reasonable in some average sense (cf. Fig. 1), and which will allow us to probe approximations (ii) and (iii) quite simply, and to compare with [1] and $[2]$.

In [1], for example, the approximation

$$
\Pi_{L} \approx \Pi_{T} \approx \frac{\alpha r}{8}+\omega_{P}^{2} \equiv \Pi^{P 1}(r, \beta)
$$

was made. On Fig. 1, this would be represented by a (zero-temperature) cone with its vertex at point $A$, for both $\Pi_{L}$ and $\Pi_{T}$. We note that Eq. (31a) is correct for both $\Pi_{L}$ and $\Pi_{T}$ as $k_{3} \rightarrow 0$, with $|k|$ set to zero [cf. (28b) and (28d)] - i.e. the $a \rightarrow \infty$ limit. With (31a), since 
both $\Pi_{L}$ and $\Pi_{T}$ remain finite as $r \rightarrow 0$ there are no infrared divergences, and both $\Pi_{L}$ and $\Pi_{T}$ can be retained. For comparison, we shall also consider the $a=0$ version of (31a), namely

$$
\Pi_{L} \approx \Pi_{T} \approx \frac{\alpha r}{8}+2 \omega_{P}^{2} \equiv \Pi^{P 2}(r, \beta) .
$$

But both (31a) and (31b) miss the flattening of the I-curves in Fig. 1 for small $r$. A somewhat better approximation to the shapes of Fig. 1, and one which was explicitly considered for $\Pi_{00}\left(=\Pi_{L}\right)$ at $k_{3}=0$ in $[2]$, is

$$
\Pi_{L} \approx \operatorname{Max}\left(\frac{\alpha r}{8}, 2 \omega_{P}^{2}\right) \equiv \Pi^{M 2}(r, \beta),
$$

which is a sawn-off cone. Equation $(32)$ is a reasonable approximation to $\Pi_{L}$ in the plane $a=0$ (instantaneous case). In the same spirit, we may consider the other limiting value

$$
\Pi_{L} \approx \operatorname{Max}\left(\frac{\alpha r}{8}, \omega_{P}^{2}\right) \equiv \Pi^{M 1}(r, \beta),
$$

which is appropriate to $a=\infty$. As mentioned in the Introduction, $\Pi_{T}$ was neglected in [2] (at $\left.k_{3}=0\right)$. Here, however, we shall keep both $\Pi_{L}$ and $\Pi_{T}$ using this "Max" form, taking

$$
\Pi_{L} \approx \Pi_{T} \approx \Pi^{M 1} \text { or } \Pi^{M 2},
$$

as our next pair of models. Again, there will be no infrared divergences. Actually, although ostensibly only $\Pi_{00}$ was retained in [2], in fact an error in Eq. (20) of that reference (the coefficient $\alpha / 2 N \pi$ should be $\alpha / 4 N \pi$ - see the footnote in Section 2.6 of [14]), means that effectively Ref. [2] considered the case $\Pi_{T}=\Pi_{L}$, so that the instantaneous limit of the $\Pi^{M 2}$ model will be directly contained in [2]. Finally, we might expect that the average effect of both $\Pi_{L}$ and $\Pi_{T}$ is best represented by

$$
\Pi_{L} \approx \Pi_{T} \approx\left[\frac{\alpha r^{2}}{64}+\omega_{P}^{4}\right]^{1 / 2} \equiv \Pi^{R 1}(r, \beta),
$$

which is a rotationally symmetric bowl with vertex at point $A$ in Fig. 1 . For completeness we also consider $\Pi^{R 2}(r, \beta)$, in which $\omega_{P}^{2}$ in $(35)$ is replaced by $2 \omega_{P}^{2}$. The three pairs of functions $\left(\Pi^{P 1}, \Pi^{P 2}\right),\left(\Pi^{M 1}, \Pi^{M 2}\right)$ and $\left(\Pi^{R 1}, \Pi^{R 2}\right)$ are the models for the self-energies which we shall use and compare in the numerical calculations which follow. We shall also introduce one more $\Pi$ in the instantaneous case.

\section{Solutions of the S-D Equation in the Instantaneous and Retarded Cases}

\subsection{The equations to be solved}

Let us denote the approximate self-energies by $\Pi^{A}$ where $A=P 1, P 2, \ldots, R 2$. Then Eq. (6) becomes

$$
1=-\frac{2 i \alpha}{N} \int \frac{d k_{0} d^{2} k}{(2 \pi)^{3}}\left\{\frac{1}{k^{2}-\Pi^{A}} \cdot \frac{1}{k^{2}-\Sigma^{2}}+\frac{2 \pi i \delta\left(k^{2}-\Sigma^{2}\right)}{\left(k^{2}-\Pi^{A}\right)\left(e^{\beta\left|k_{0}\right|}+1\right)}\right.
$$




$$
\left.-\frac{2 \pi i \delta\left(k^{2}-\Pi^{A}\right)}{\left(k^{2}-\Sigma^{2}\right)\left(e^{\beta\left|k_{0}\right|}-1\right)}\right\}
$$

where $k^{2}=k_{0}^{2}-k^{2}$. We shall define the instantaneous approximation by

$$
k^{2}-\Pi^{A}(r, \beta) \approx-\left(k^{2}+\Pi^{A}(|k|, \beta)\right) \equiv-\left(k^{2}+\Pi_{\text {inst }}^{A}(|k|, \beta)\right) .
$$

We begin by arguing that the contribution from the third term in the braces in (36) is negligible. Consider first the instantaneous case. Using the $\delta$-function to do the $k_{0}$-integration, the third term in (36) becomes

$$
-\frac{\alpha}{\pi N} \int_{0}^{\infty} \frac{K d K}{E\left(\Pi_{i n s t}^{A}\right)} \cdot \frac{1}{\Pi_{i n s t}^{A}-\Sigma^{2}} \cdot \frac{1}{\exp \left[\beta E\left(\Pi_{i n s t}^{A}\right)\right]-1}
$$

where $K=|k|$ and

$$
E^{2}\left(\Pi_{i n s t}^{A}\right)=K^{2}+\Pi_{i n s t}^{A} .
$$

To estimate the magnitude of (38), we initially neglect this term altogether, and solve the remaining equation (in the instantaneous approximation), as described below. This produces a solution $\Sigma=\Sigma_{\text {inst }}^{A}(T)$, say, for $0 \leq T \leq T_{c, \text { inst }}$. We then evaluate (38) for typical $\Sigma$ and $T$ values, on the curve $\Sigma=\Sigma_{\text {inst }}^{A}(T)$. For all the $\Pi_{\text {inst }}^{A}$ 's we found that (38) was typically of order $10^{-4}$ or less, thus verifying the consistency of neglecting this term.

In the non-instantaneous case, we choose $\Pi^{R}$ as a representative $\Pi$. The $\delta$-function in the third term in (36) is then (returning to Minkowski space)

$$
\delta\left(k_{0}^{2}-k^{2}-\left[\omega_{P}^{4}-\frac{\alpha^{2}}{64}\left(k_{0}^{2}-k^{2}\right]^{1 / 2}\right) .\right.
$$

Again, neglecting this third term altogether (see below) we find a solution $\Sigma=\Sigma_{\text {ret }}^{R}(T)$, say. This tells us the order of magnitude of the typical temperatures of interest, $T \lesssim T_{c, \text { ret }}$. These temperatures are such that $\omega_{P}^{2} \ll \alpha / 8$. Returning to $(40)$, the root of the $\delta$-function is then given approximately by

$$
k_{0}^{2}=k^{2}+\frac{64 \omega_{P}^{4}}{\alpha^{2}} .
$$

But this means that the $\delta$-function is effectively

$$
\delta\left(\left[\omega_{P}^{4}-\frac{\alpha^{2}}{64}\left(k_{0}^{2}-k^{2}\right)\right]^{1 / 2}\right)
$$

which gives zero if (as in this case) the quantity multiplying (42) is finite at the point (41). We conclude that this term is negligibly small. We note that it was omitted entirely in [1], and that the analogous term in [8] is also numerically very small. 
We are left with just the first two terms of (36). Consider first the instantaneous case. The $k_{0}$-integration in the first term can be done by contour integration with the result

$$
I_{\text {inst }}^{(1)}(\Sigma, \beta)=\frac{\alpha}{2 \pi N} \int \frac{K d K}{E_{\Sigma}(K)} \frac{1}{K^{2}+\prod_{i n s t}^{A}},
$$

where $E_{\Sigma}(K)=\sqrt{K^{2}+\Sigma^{2}}$. In the second term the $k_{0}$-integration is done by the $\delta$-function, leading to

$$
I_{\text {inst }}^{(2)}(\Sigma, \beta)=-\frac{\alpha}{\pi N} \int \frac{K d K}{E_{\Sigma}(K)} \quad \frac{1}{K^{2}+\prod_{\text {inst }}^{A}} \quad \frac{1}{e^{\beta E_{\Sigma}}+1} .
$$

The S-D equation in the instantaneous case is then

$$
1=\frac{\alpha}{2 N \pi} \int_{0}^{\infty} \frac{K d K}{E_{\Sigma}(K)} \quad \frac{1}{K^{2}+\prod_{i n s t}^{A}(K, \beta)} \quad \tanh \left(\beta E_{\Sigma}(K) / 2\right) .
$$

Equation (45) is the same as Eq. (20) of [2], interpreting $\Pi_{i n s t}^{A}$ as $K^{2} \Pi_{00}$ of that reference. It is of the standard "gap equation" type, and may also be compared with Eq. (17) of [5], for instance. We remark that we have set the upper limit of the integration in (45) as infinity: it would of course be possible to consider the effects of a finite cut-off (for example $\Lambda=\alpha$ ), but this was carefully studied in [2] and need not be repeated here. In fact, the solution was found [2] to be relatively insensitive to the value of $\Lambda$, and for simplicity we shall take $\Lambda \rightarrow \infty$ as in (45), which is included in [2].

We now turn to the retarded (non-instantaneous) case. The first term in (36) is straightforward: rotating into Euclidean space via $k_{0} \rightarrow i k_{3}$ it becomes

$$
I_{r e t}^{(1)}(\Sigma, \beta)=\frac{\alpha}{N \pi^{2}} \int \frac{r^{2} d r}{r^{2}+\Pi^{A}(r, \beta)} \frac{1}{r^{2}+\Sigma^{2}} .
$$

We remark that for $A=P$ and $M$ the integrals (46) can be easily performed in terms of elementary functions, but we shall not list the results here. With the second term we encounter a difficulty, however. Doing the $k_{0}$-integration by the $\delta$-function, we need to evaluate $\Pi^{A}$ at the point $k^{2}=k_{0}^{2}-k^{2}=\Sigma^{2}$ in Minkowski space. Now the $\Pi^{A}$ 's of (31) and (34) are written in Euclidean space, and when continued back to Minkowski space they will involve the quantity $\sqrt{k^{2}-k_{0}^{2}}=\sqrt{-k^{2}}$, which is imaginary at $k^{2}=\Sigma^{2}$. The same will be true for the $\Pi^{R}$ functions at sufficiently small (non-zero) $T$, and would also happen if we used the exact self energies (26) and (27). This is an unphysical result, and has arisen because we have evaluated the vacuum polarization for massless fermions (recall that $\alpha \sqrt{-k^{2}} / 8$ is the $\Sigma \rightarrow 0$ limit of the zero-temperature self-energies $\Pi_{L}(k, \beta=\infty)=\Pi_{T}(k, \beta=\infty)=\Pi\left(k^{2}\right)$ - see (14), (15), (18) and (19)). The point $k^{2}=\Sigma^{2}$ is above the threshold $k^{2}=0$ for the production of a real massless fermion-antifermion pair, which is why $\Pi^{P}$ and $\Pi^{M}$, for example, are complex at $k^{2}=\Sigma^{2}$. In fact, however, if the fermion mass $\Sigma$ were retained in the vacuum polarization, the threshold would be at $k^{2}=4 \Sigma^{2}$ [c.f. (18)] and $k^{2}=\Sigma^{2}$ would be below it so that the exact $\Pi$ 's in that case would be real.

We note, however, that the second term in (36) is well defined in the limit $\Sigma \rightarrow 0$, namely

$$
\left.\begin{array}{rlll}
I_{\text {ret }}^{(2)}(\Sigma \rightarrow 0, \beta) & =-\frac{1}{N} & \text { for } & A=P 1, M 1, R 1 \\
& =-\frac{1}{2 N} & \text { for } & A=P 2, M 2, R 2,
\end{array}\right\}
$$


while of course

$$
I_{\text {ret }}^{(2)}(\Sigma, \beta \rightarrow \infty)=0 .
$$

The limits (47) and (48) are in fact all that is needed to discuss the ratio $r=2 \Sigma(T=0) / k_{B} T_{c}$, since $T_{c}$ is determined from the solution of (36) as $\Sigma \rightarrow 0$, for which (47) is required, while $\Sigma(T=0)$ uses $(48)$. The $\Sigma \rightarrow 0$ limit of the second term in (36) was also calculated in [1], but the value given there appears to have the wrong sign, and was in any case neglected on the ground that it is of $O(1 / N)$ as compared with the first term, which must be $O(1)$. This is actually a dangerous argument, for the following reason. The solution $\Sigma$ depends roughly exponentially on $N$, as at zero temperature [15]:

$$
\Sigma \sim \exp -b N
$$

and an $O(1 / N)$ term on the right-hand side of (36), when taken to the left-hand side, becomes an $O(1 / N)$ correction to $b$ in $(49)$, and hence an $O(1)$ effect in $\Sigma$.

To obtain $\Sigma$ as a function of $T$, however, we need an approximation for $I_{\text {ret }}^{(2)}$ which interpolates sensibly between (47) and (48). Since the dynamically generated mass $\Sigma$ is in fact always small, a reasonable approximation is to replace $\Pi^{A}\left(k^{2}, \beta\right)$ in $I_{\text {ret }}^{(2)}$ by its value at $k^{2}=0$, namely $(1$ or 2) $\omega_{P}^{2}$. The second term in $(36)$ then becomes

$$
\left.\begin{array}{rl}
I_{\text {ret }}^{(2)}(\Sigma, \beta) & =\frac{\alpha k_{B} T}{\pi N\left(\Sigma^{2}-\omega_{P}^{2}\right)} \ln \left(1+e^{-\beta \Sigma}\right) \quad \text { for } \quad A=P 1, M 1, R 1 \\
& =\frac{\alpha k_{B} T}{\Pi N\left(\Sigma^{2}-2 \omega_{P}^{2}\right)} \ln \left(1+e^{-\beta \Sigma}\right) \quad \text { for } \quad A=P 2, M 2, R 2 .
\end{array}\right\}
$$

Our S-D equation in the retarded case is therefore

$$
1=I_{\text {ret }}^{(1)}(\Sigma, \beta)+I_{\text {ret }}^{(2)}(\Sigma, \beta) .
$$

Eqs. (45) and (51) define the models we have solved numerically. We have also considered one more case (also discussed in [2]), that in which $\Pi_{\text {inst }}^{A}$ in (45) is replaced by its zero-temperature limit $\alpha K / 8$, denoted by

$$
\Pi_{\text {inst }}^{Z} \equiv \Pi_{\text {inst }}^{A}(K, \infty)=\alpha \frac{K}{8} .
$$

Results using this function are expected to be closer to BCS values, where the analogous kernel is approximately temperature-independent. (We cannot consider $\Pi^{A}=\Pi^{A}(\beta=\infty)$ in the retarded case, as the integral in $I_{\text {ret }}^{(2)}$ would then diverge as $\Sigma \rightarrow 0$; this divergence would be cured by allowing $\Sigma$ to depend on momentum.)

\subsection{Numerical Results, and Conclusions}

Results for the instantaneous case are presented in Fig. 2 and in Tables $1 \mathrm{a}$ and $1 \mathrm{~b}$. Figure 2 shows, for $N=2$ the solutions $\Sigma$ of Eq. (45) versus $T$ (both in units of $\alpha$ ), for the different $\Pi_{\text {inst }}^{A}$ 's $(A=P 1, \ldots, Z)$. Table 1 a lists, for $N=1$ to 5 and $A=(P 1, R 1, M 1, Z)$, the values of $s_{0} \equiv \Sigma(T=0) / \alpha, t_{c} \equiv k_{B} T_{c} / \alpha$ and $r \equiv 2 s_{0} / t_{c}$, where $T_{c}$ is the temperature above which $\Sigma=0$. 
Table $1 \mathrm{~b}$ gives the similar list for $A=P 2, R 2$ and $M 2$. The corresponding retarded results are shown in Fig. 3 (also for $N=2$ ) and in Tables 2 a and $2 \mathrm{~b}$.

In discussing these results, we shall want to distinguish the effects of - on the one hand using the instantaneous versus the retarded self-energies $\Pi^{A}$, and - on the other - the different choices $A=P 1, P 2$, etc. With this in mind, there are some simple qualitative features of the results which we can identify, as follows.

i) The values of $s_{0}$ and $t_{c}$ for a given $N$ and $A$ are, very roughly, an order of magnitude smaller in the retarded than in the instantaneous case.

ii) For the choices $A=P 2, R 2$ and $M 2$, the $r$ values for a given $N$ are smaller in the retarded than in the instantaneous case (compare Tables $1 \mathrm{~b}$ and $2 \mathrm{~b}$ ). The same is true for $A=M 1$, but not always for $A=R 1$, and the reverse is true for $A=P 1$ (Tables 1 a and $2 \mathrm{a}$ ). Nevertheless, it is broadly true that retardation reduces $r$.

iiia) In the instantaneous case, the $\Sigma-T$ curve for " $A=X 1$ " is always to the right of the curve for " $A=X 2$ ", so that $r$ for each of $P 1, R 1$ and $M 1$ is always significantly less than for each of $P 2, R 2$ and $M 2$, respectively. The same is seen from a comparison of the $r$-columns in Tables $1 \mathrm{a}$ and $1 \mathrm{~b}$.

iiib) In the retarded case, there is much less difference between the " 1 " and " 2 " alternatives, especially as concerning the $r$-values. A few of the $r$ values (at small $N$ ) for " 1 " are actually larger than those for " 2 ".

iv) There is a similar ordering of the curves, and hence of the $r$ values, according to the alternatives $A=P, R$ and $M$. This is especially clear in the retarded case. For example, Fig. 3 shows that the $\Sigma-T$ curves for " $M$ " are to the right of those for " $R$ ", which in turn are to the right of those for " $P$ ". This means in particular than $r(M 1$ or $M 2)<r(R 1$ or $R 2)<r(P 1$ or $P 2)$ (in an obvious notation), which is also true of all entries in Tables $2 \mathrm{a}$ and $2 \mathrm{~b}$, for a given $N$. In the instantaneous case, there is more overlap caused by the 1-2 alternatives, but it is still true in all cases (see also Tables $1 \mathrm{a}$ and $1 \mathrm{~b}$ ) that $r(M 1)<r(R 1)<R(P 1)$, and that $r(M 2)<r(R 2)<r(P 2)$.

v) The "rightmost" curve, by a significant margin, is the instantaneous " $A=Z$ " case (zerotemperature limit of $\left.\Pi_{i n s t}^{A}\right)$; correspondingly the $r$-values for this case are the smallest (column $Z$ of Table 1a).

On the basis of (iii) $-(\mathrm{v})$ above we can make an important broad generalization: the value of $r$ is closely correlated with what we might call "the average height of $\Pi$ above the origin", in Fig. 1. This is determined partly by the intercept of the vertical axis, and partly by the degree of "flatness" of $\Pi$ for small $r$. Thus the smallest $r$ is found for $\Pi_{\text {inst }}^{Z}$, which passes through the origin. The $\Pi^{M}$ 's are flatter than the $\Pi^{R}$ s, which in turn are flatter than the $\Pi^{P}$ 's, and the $r^{\prime}$ 's increase correspondingly. Generally, the $r$ 's for $\Pi^{1}$ 's are less than those for $\Pi^{2}$ 's. This observation is, in a sense, a refinement of the one made in [2]: that the large $r$-value found there was attributable to the thermal screening displayed in Eq. (1). A similar conclusion can be 
drawn from the results of [8], which imply (for QCD in four dimensions) the value $r \approx 3.51$ using a zero-temperature gluon self-energy, and $r \approx 9.1$ for a thermally screened gluon propagator.

This brings us to a comparison of our results with those of [2]. Table 4 of that reference shows the results of solving Eq. (45) (= Eq. (20) of [2]) for $N=1-5$, with the cut-off $\Lambda \rightarrow \infty$, and using the self-energy $\Pi_{i n s t}^{M 2}$. These should correspond to the $M 2$ column of our Table 1b. Table 4 of [2] also lists the $r$ values for the $\Pi_{\text {inst }}^{Z}$ model, which are given in column $Z$ of our Table 1a. Unfortunately, there is not perfect agreement between the two sets of results, although they are generally quite close. We might just remark that the $r$-values of [2] vary somewhat irregularly with $N$, which is surprising -whereas ours behave quite smoothly.

What can one conclude from this study, as regards the value of $r$ ? The values found in [2] (or Table $1 \mathrm{~b}$ ) using $\Pi_{\text {inst }}^{M 2}(r \approx 8-9)$ are likely to be too high, because (a) retardation reduces $r$ and (b) $\Pi^{M 2}$ is too "high" on Fig. 1 to represent a fair average $\Pi$. In the (instantaneous) plane $a=0$, a more reasonable average of $\Pi_{L}$ and $\Pi_{T}$ would seem to be the choice $M 1$, rather than M2. From Table 1a, this would imply r's of about 6 , in this range of $N$, for the instantaneous case. On the other hand, the $\Pi^{M}$ 's are too flat near $r=0$, and the $\Pi^{R}$ 's (which are a better fit to the shape of the exact $\Pi^{\prime}$ 's) give somewhat larger $r$ 's than the corresponding $\Pi^{M}$ 's: for example, $\Pi_{\text {inst }}^{R 1}$ leads to $r \approx 7$. Including retardation takes us to the case $\Pi^{R 1}$ (which seems to be the best "average $\Pi$ "), and (from Table $2 \mathrm{a}$ ) a value of $r \approx 6$ for $N$ in the range 2-4. The value $N=2$ is appropriate to the superconductivity application [14], and $r \approx 6$ is still considerably larger than the BCS value of about 3.5.

The calculations reported above are, of course, capable of considerable improvement. It would be interesting to see the effect of using the approximations (29) and (30), for example, which include the $a$-dependence (lack of rotational invariance in $k_{3}-|k|$ ) present in the exact $\Pi$ 's. These more realistic self-energies should be combined with a momentum-dependent mass, and with wave function renormalization, to give a picture of dynamical mass generation in $\mathrm{QED}_{3}$ at finite temperature, free of all the approximations (ii)-(v) of the Introduction.

Acknowledgements I am grateful to César Fosco and Nick Mavromatos for reading the manuscript,and for making useful comments and suggestions. 
The zero-temperature fermion mass $\Sigma(T=0)$ in units of $\alpha\left(s_{0}=\Sigma(T=0) / \alpha\right)$ and the critical temperature $T_{c}$ in units of $\alpha\left(t_{c}=T_{c} / \alpha\right)$, together with the ratio $r=2 s_{0} / t_{c}$, for $N=1,2,3,4,5$, calculated in the instantaneous approximation using the self-energies $\prod_{\text {inst }}^{A}$ with $A=P 1, R 1, M 1$ and $Z$.

\begin{tabular}{|c|l||c|c||c|c||c|c||c|c||}
\cline { 3 - 10 } \multicolumn{2}{l|}{} & \multicolumn{2}{c||}{$\mathrm{P} 2$} & \multicolumn{2}{c||}{$\mathrm{R} 2$} & \multicolumn{2}{c||}{ M2 } \\
\hline $\mathrm{N}$ & \multicolumn{1}{c|}{$s_{0}$} & $t_{c}$ & $r$ & $t_{c}$ & $r$ & $t_{c}$ & $r$ & $t_{c}$ & $r$ \\
\hline 1 & 0.293 & $6.7 \times 10^{-2}$ & 8.75 & $8.31 \times 10^{-2}$ & 7.05 & $9.14 \times 10^{-2}$ & 6.41 & 0.156 & 4.13 \\
\hline 2 & $7.68 \times 10^{-2}$ & $1.6 \times 10^{-2}$ & 9.60 & $2.22 \times 10^{-2}$ & 6.92 & $2.6 \times 10^{-2}$ & 5.91 & $4.18 \times 10^{-2}$ & 3.68 \\
\hline 3 & $2.83 \times 10^{-2}$ & $5.47 \times 10^{-3}$ & 10.35 & $8.26 \times 10^{-3}$ & 6.85 & $1 \times 10^{-2}$ & 5.66 & $1.57 \times 10^{-2}$ & 3.61 \\
\hline 4 & $1.17 \times 10^{-2}$ & $2.15 \times 10^{-3}$ & 10.88 & $3.44 \times 10^{-3}$ & 6.80 & $4.24 \times 10^{-3}$ & 5.52 & $6.56 \times 10^{-3}$ & 3.57 \\
\hline 5 & $5.1 \times 10^{-3}$ & $9.07 \times 10^{-4}$ & 11.25 & $1.51 \times 10^{-3}$ & 6.75 & $1.87 \times 10^{-3}$ & 5.45 & $2.88 \times 10^{-3}$ & 3.54 \\
\hline
\end{tabular}

Table $1 \mathrm{~b}$

The mass $s_{0}$, temperature $t_{c}$, and ratio $r$ (as in Table $1 \mathrm{a}$ ) for $N=1,2,3,4,5$ calculated in the instantaneous approximation using the self-energies $\Pi_{\text {inst }}^{A}$ with $A=P 2, R 2, M 2$.

\begin{tabular}{|c|l||c|c||c|c||c|c||}
\cline { 2 - 7 } \multicolumn{2}{l||}{} & \multicolumn{2}{c||}{$\mathrm{P} 2$} & \multicolumn{2}{c||}{$\mathrm{R} 2$} & \multicolumn{2}{c||}{ M2 } \\
\hline $\mathrm{N}$ & \multicolumn{1}{c||}{$s_{0}$} & $t_{c}$ & $r$ & $t_{c}$ & $r$ & $t_{c}$ & $r$ \\
\hline 1 & 0.293 & $4.88 \times 10^{-2}$ & 12.01 & $6.16 \times 10^{-2}$ & 9.51 & $6.77 \times 10^{-2}$ & 8.66 \\
\hline 2 & $7.68 \times 10^{-2}$ & $1.08 \times 10^{-2}$ & 14.22 & $1.56 \times 10^{-2}$ & 9.85 & $1.86 \times 10^{-2}$ & 8.26 \\
\hline 3 & $2.83 \times 10^{-2}$ & $3.56 \times 10^{-3}$ & 15.90 & $5.66 \times 10^{-3}$ & 10.00 & $7.07 \times 10^{-3}$ & 8.01 \\
\hline 4 & $1.17 \times 10^{-2}$ & $1.37 \times 10^{-3}$ & 17.08 & $2.33 \times 10^{-3}$ & 10.04 & $2.99 \times 10^{-3}$ & 7.83 \\
\hline 5 & $5.1 \times 10^{-3}$ & $5.71 \times 10^{-4}$ & 17.86 & $1.01 \times 10^{-3}$ & 10.10 & $1.32 \times 10^{-3}$ & 7.73 \\
\hline
\end{tabular}

Table $2 \mathrm{a}$

The mass $s_{0}$, temperature $t_{c}$, and ratio $r$, for $N=1,2,3,4,5$, calculated including retardation and using the self-energies $\Pi^{A}$ with $A=P 1, R 1, M 1$.

\begin{tabular}{|c|c||c|c||c|c||c|c||}
\cline { 3 - 8 } \multicolumn{2}{l||}{} & \multicolumn{2}{c||}{$\mathrm{P} 1$} & \multicolumn{2}{c||}{$\mathrm{R} 1$} & \multicolumn{2}{c||}{ M1 } \\
\hline $\mathrm{N}$ & $s_{0}$ & $t_{c}$ & $r$ & $t_{c}$ & $r$ & $t_{c}$ & $r$ \\
\hline 1 & $5.78 \times 10^{-2}$ & $9.16 \times 10^{-3}$ & 12.62 & $1.52 \times 10^{-2}$ & 7.61 & $1.92 \times 10^{-2}$ & 6.02 \\
\hline 2 & $1.205 \times 10^{-2}$ & $2.05 \times 10^{-3}$ & 11.76 & $3.76 \times 10^{-3}$ & 6.41 & $4.98 \times 10^{-3}$ & 4.84 \\
\hline 3 & $3.21 \times 10^{-3}$ & $5.41 \times 10^{-4}$ & 11.87 & $1.04 \times 10^{-3}$ & 6.17 & $1.40 \times 10^{-3}$ & 4.59 \\
\hline 4 & $9.09 \times 10^{-4}$ & $1.52 \times 10^{-4}$ & 11.96 & $2.99 \times 10^{-4}$ & 6.08 & $4.05 \times 10^{-4}$ & 4.49 \\
\hline 5 & $2.62 \times 10^{-4}$ & $4.36 \times 10^{-5}$ & 12.02 & $8.66 \times 10^{-5}$ & 6.05 & $1.18 \times 10^{-4}$ & 4.44 \\
\hline
\end{tabular}


Table 2b

As in Table 2a, using the self-energies $\Pi^{A}$ with $A=P 2, R 2, M 2$.

\begin{tabular}{|c|c||c|c||c|c||c|c||}
\cline { 3 - 8 } \multicolumn{2}{l|}{} & \multicolumn{2}{c||}{ P2 } & \multicolumn{2}{c||}{ R2 } & \multicolumn{2}{c||}{ M2 } \\
\hline $\mathrm{N}$ & $s_{0}$ & $t_{c}$ & $r$ & $t_{c}$ & $r$ & $t_{c}$ & $r$ \\
\hline 1 & $5.78 \times 10^{-2}$ & $1.11 \times 10^{-2}$ & 10.41 & $1.72 \times 10^{-2}$ & 6.72 & $2.07 \times 10^{-2}$ & 5.58 \\
\hline 2 & $1.205 \times 10^{-2}$ & $2.10 \times 10^{-3}$ & 11.48 & $3.70 \times 10^{-3}$ & 6.51 & $4.80 \times 10^{-3}$ & 5.02 \\
\hline 3 & $3.21 \times 10^{-3}$ & $5.21 \times 10^{-4}$ & 12.32 & $9.82 \times 10^{-4}$ & 6.54 & $1.31 \times 10^{-3}$ & 4.90 \\
\hline 4 & $9.09 \times 10^{-4}$ & $1.43 \times 10^{-4}$ & 12.71 & $2.78 \times 10^{-4}$ & 6.54 & $3.76 \times 10^{-4}$ & 4.84 \\
\hline 5 & $2.62 \times 10^{-4}$ & $4.06 \times 10^{-5}$ & 12.91 & $8.03 \times 10^{-5}$ & 6.53 & $1.09 \times 10^{-4}$ & 4.81 \\
\hline
\end{tabular}




\section{References}

[1] A. Kocić, Phys.Lett. B189 (1987) 449.

[2] N. Dorey and N.E. Mavromatos, Phys.Lett. B2566 (1991) 163.

[3] I.J.R. Aitchison, N. Dorey, M. Klein-Kreisler and N.E. Mavromatos, Phys.Lett. B294 (1992) 91.

[4] I.J.R. Aitchison and M. Klein-Kreisler, Phys.Rev. D50 (1994) 1068.

[5] D. Bailin, J. Cleymans and M.D. Scadron, Phys.Rev. D31 (1985) 164.

[6] A.C. Davis and A.M. Matheson, Nucl.Phys. B246 (1984) 203.

[7] A.C. Davis and A.M. Matheson, Phys.Rev. D40 (1989) 2373.

[8] T.S. Evans and R.J. Rivers, Z.Phys. C40 (1988) 293.

[9] T.W. Appelquist, D. Nash and L.C. Wijewardhana, Phys.Rev.Lett. 60 (1988) 2575.

[10] M.R. Pennington and S.P. Webb, BNL Report No. BNL-40886 (1988) (unpublished);

D. Atkinson, P.W. Johnson and M.R. Pennington, BNL Report No BNL-41615 (1988) (unpublished);

M.R. Pennington and D. Walsh, Phys.Lett. B253 (1991) 246;

D.C. Curtis, M.R. Pennington and D. Walsh, Phys.Lett. B295 (1992) 313;

K.-I. Kondo and H. Nakatani, Progr.Theor.Phys. 87 (1992) 193.

[11] M. Crisan, Theory of Superconductivity (World Scientific, Singapore(1988)).

[12] B.Batlogg in Proceedings of the Los Alamos Symposium on High Temperature Superconductivity,Eds.K.S.Bedell,D.Coffey, D.E.Meltzer,D.Pines and J.R.Schrieffer (Addison Wesley, Reading,MA (1990)).

[13] A. Kovner and B. Rosenstein, Phys.Rev. B42 (1990) 4748;

N. Dorey and N.E. Mavromatos, Phys.Lett. B250 (1990) 107;

G.W. Semenoff and N. Weiss, Phys.Lett. B250 (1990) 117.

[14] N. Dorey and N.E. Mavromatos, Nucl.Phys. B386 (1992) 614.

[15] R.D. Pisarski, Phys.Rev. D29 (1984) 2423.

[16] T.W. Appelquist, M. Bowick, D. Karabali and L.C.R. Wijewardhana, Phys.Rev. D33 (1986) 3704;

E. Dagotto, A. Kocić and J.B. Kogut, Phys.Rev.Lett. 62 (1989) 1083 and Nucl.Phys. B334 (1990) 279;

D. Nash, Phys.Rev.Lett. 62 (1989) 3024;

D. Atkinson, P.W. Johnson and P. Maris, Phys.Rev. D42 (1990) 602. 
[17] N.P. Landsman and C.G. van Weert, Physics Reports 145 (1987) 141.

[18] R. Kobes and G.W. Semenoff, Nucl.Phys. B260 (1985) 714; B272 (1988) 329.

[19] L. Dolan and R. Jackiw, Phys.Rev. D9 (1974) 3320.

[20] H.A. Weldon, Phys.Rev. D26 (1982) 1394.

[21] M.R. Pennington and D. Walsh, Phys.Lett. 253B (1991) 246.

[22] I.J.R. Aitchison and J.A. Zuk, "The Non-Local Odd-Parity $O\left(e^{2}\right)$ Effective Action of QED 3 at Finite Temperature", CERN Preprint TH. 7394/94 (1994).

[23] H.A. Weldon, Phys.Rev. D47 (1993) 594.

\section{FIGURE CAPTIONS}

Fig. 1 The longitudinal $\left(\Pi_{L}\right)$ and transverse $\left(\Pi_{T}\right)$ photon self-energies as functions of $|k|$ and $k_{3}$. Three planar sections are shown: $a=0\left(k_{3}=0\right), a=1 / 2\left(k_{3}=|k| / 2\right)$ and $a=\infty(|k|=$ $0)$. $\Pi_{L}$ and $\Pi_{T}$ are represented by the height above the point $\left(|k|, k_{3}\right)$. OA has the value $\omega_{P}^{2}$, OB the value $2 \omega_{P}^{2}$. The straight lines at roughly $45^{0}$ represent the "zero-temperature cone", whose height is given by $\alpha \sqrt{k^{2}+k_{3}^{2}} / 8$.

Fig. 2 Fermion mass $\Sigma$ versus temperature $T$ (both in units of $\alpha$ ) obtained in the instantaneous approximation by solving Eq. (45) with the various $\prod_{i n s t}^{A}$ functions $(A=P 1, \ldots Z)$.

Fig. 3 Fermion mass $\Sigma$ versus temperature $T$ (both in units of $\alpha$ ) obtained including retardation by solving Eq. (51) with the various $\Pi^{A}$ functions $(A=P 1, \ldots, M 2)$. 

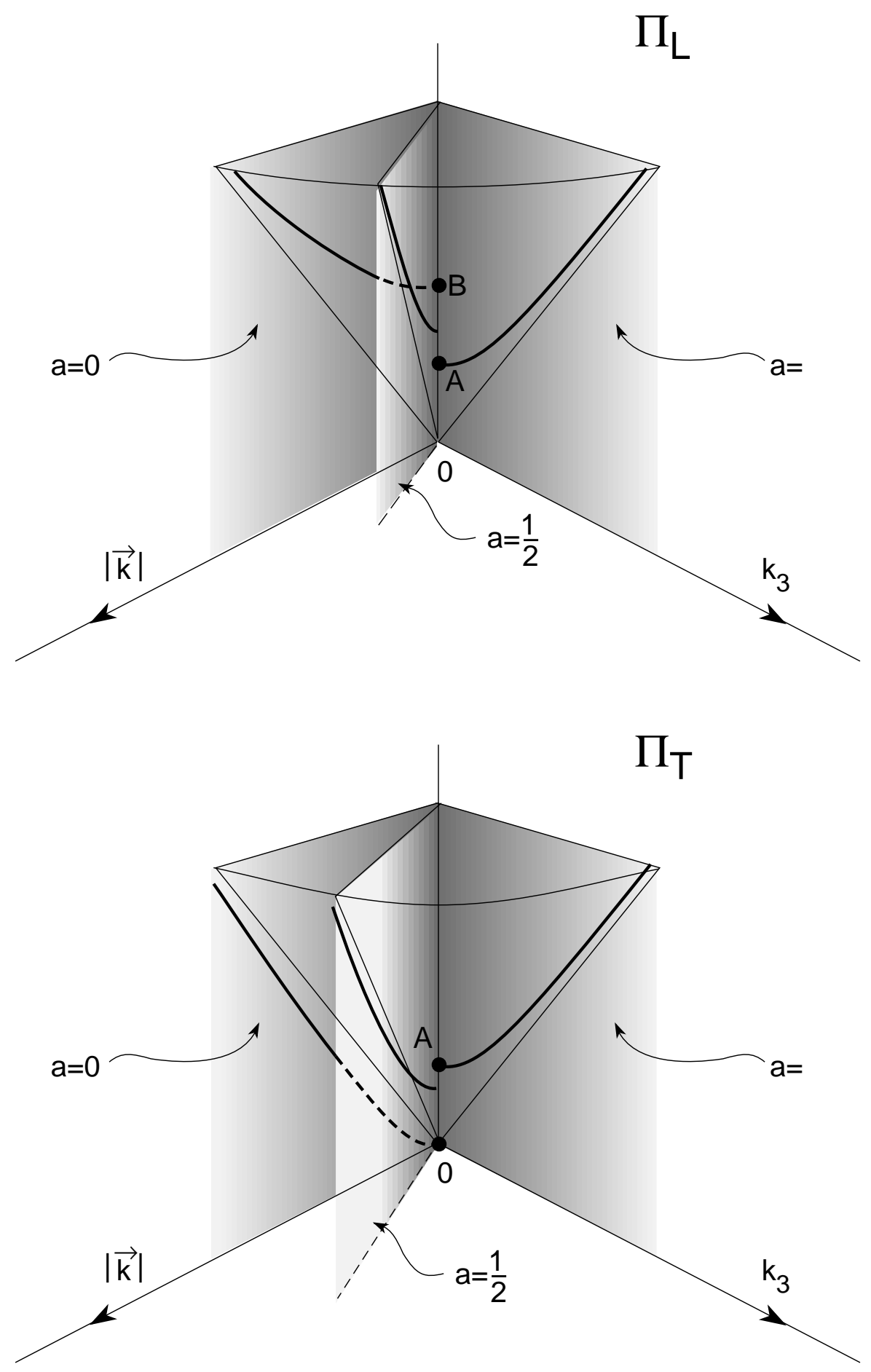

Figure 1 


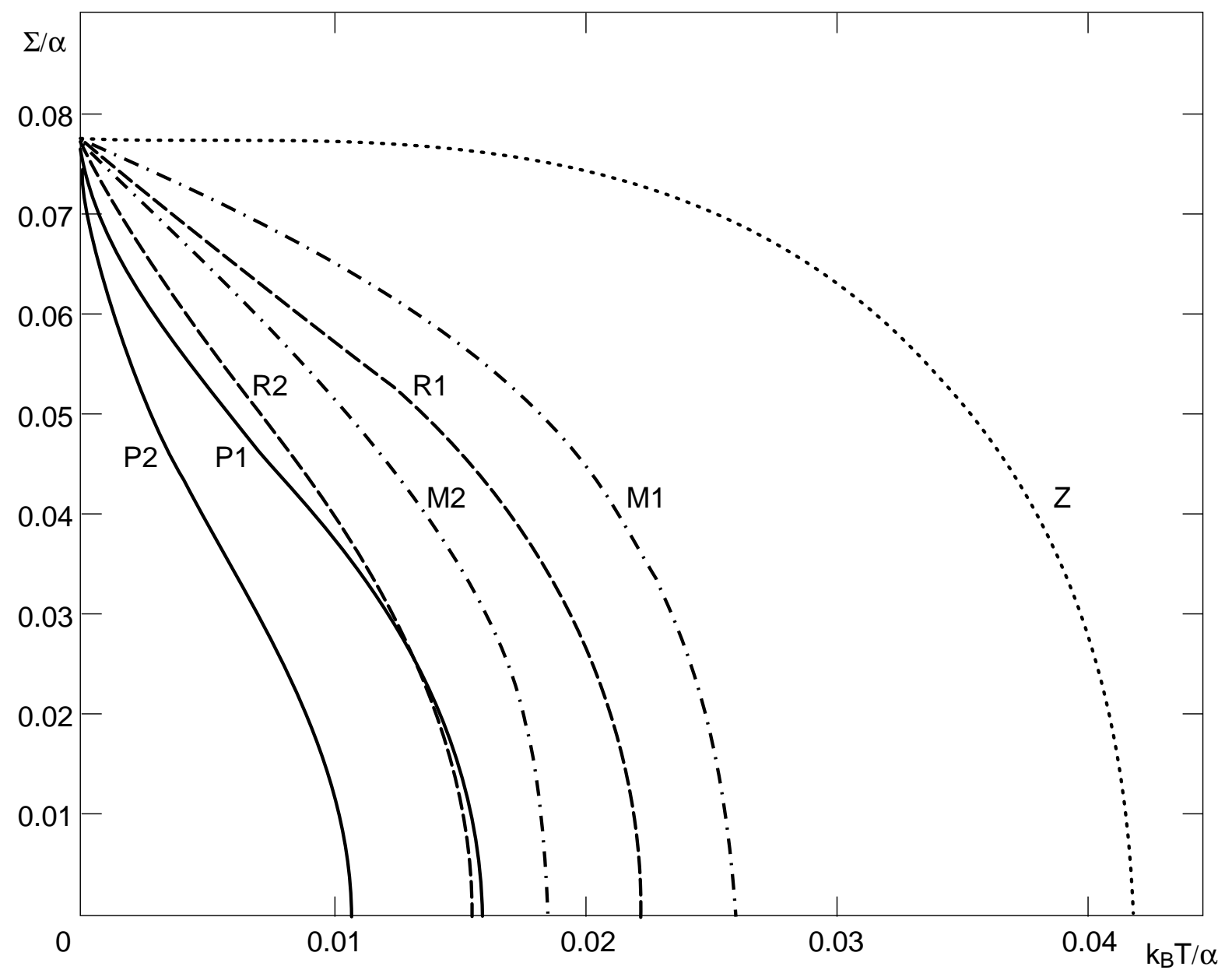

Figure 2 


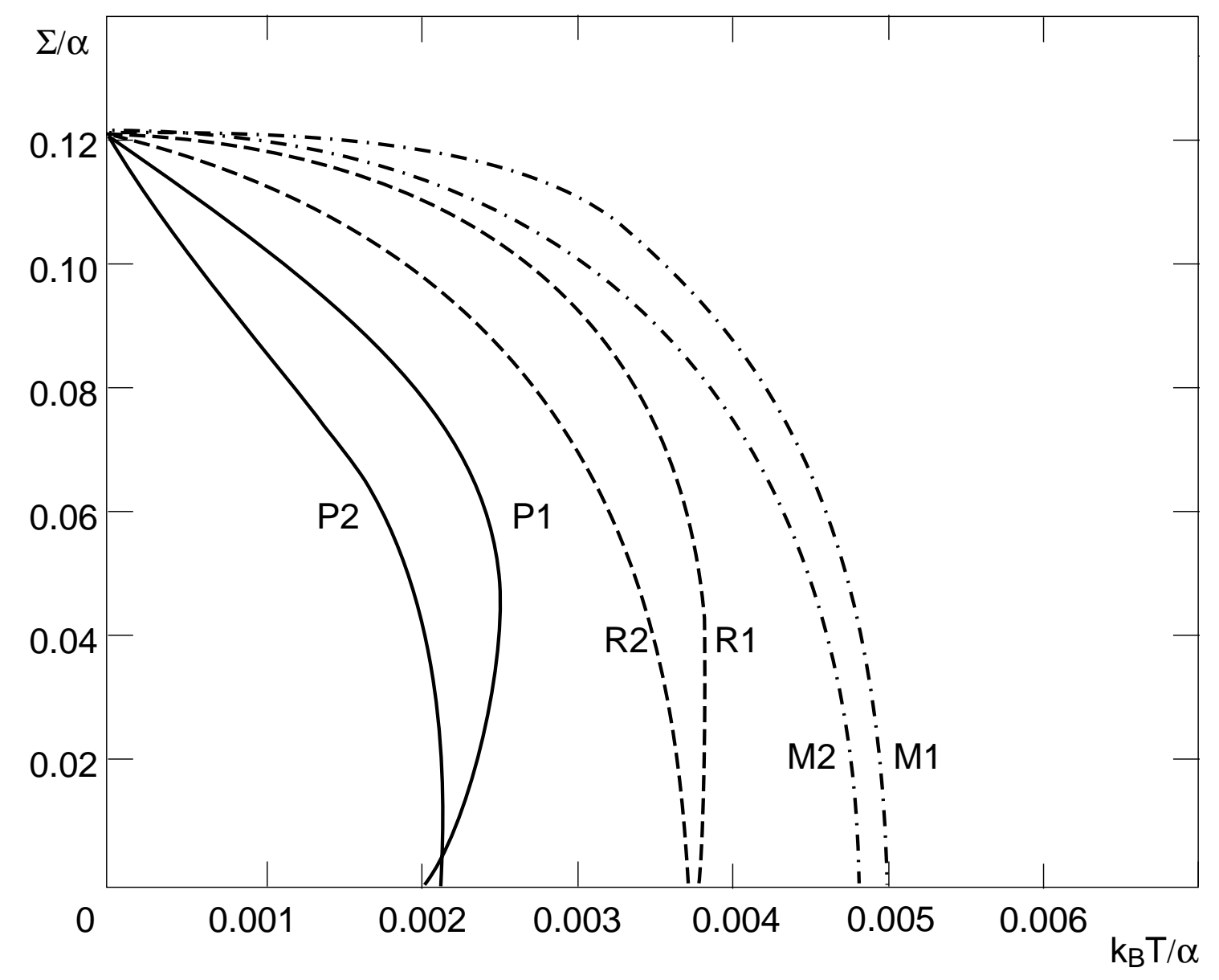

Figure 3 